Research Article

\title{
Two Pathogenic Gene Mutations Identified Associating with Congenital Cataract and Iris Coloboma Respectively in a Chinese Family
}

\author{
Bin Li $\mathbb{D}^{1,2}$ Bin Lu ${ }^{1},{ }^{3}$ Xuewen Guo, ${ }^{4}$ Shenghui Hu, ${ }^{5}$ Guihu Zhao, ${ }^{1}$ Weihong Huang, \\ Jianzhong $\mathrm{Hu} \mathbb{D}^{1},{ }^{1,2,6}$ and Kun Song $\mathbb{D}^{7}$ \\ ${ }^{1}$ National Clinical Research Center for Geriatric Disorders, Xiangya Hospital, Central South University, Changsha, China \\ ${ }^{2}$ Mobile Health Ministry of Education-China Mobile Joint Laboratory, Xiangya Hospital, Central South University, \\ Changsha, China \\ ${ }^{3}$ Department of Pathogen Biology, School of Basic Medical Sciences, Central South University, Changsha, China \\ ${ }^{4}$ Department of Neurology, Liaocheng Dongchangfu People's Hospital, Liaocheng, China \\ ${ }^{5}$ Department of Orthopaedics, Xiangya Second Hospital, Central South University, Changsha, China \\ ${ }^{6}$ Department of Spine Surgery, Xiangya Hospital, Central South University, Changsha, China \\ ${ }^{7}$ Department of Gastrointestinal Surgery, Xiangya Hospital, Central South University, Changsha, China
}

Correspondence should be addressed to Jianzhong Hu; jianzhonghu@hotmail.com and Kun Song; sk-zorro@163.com

Received 5 July 2019; Accepted 26 December 2019; Published 19 February 2020

Academic Editor: Alessandro Meduri

Copyright $\odot 2020$ Bin Li et al. This is an open access article distributed under the Creative Commons Attribution License, which permits unrestricted use, distribution, and reproduction in any medium, provided the original work is properly cited.

Purpose. To screen out pathogenic genes in a Chinese family with congenital cataract and iris coloboma. Material and Methods. A three-generation family with congenital cataract and iris coloboma from a Han ethnicity was recruited. DNA was extracted from peripheral blood samples collected from all individuals in the family. Whole exon sequencing was employed for screening the disease-causing gene mutations in the proband, and Sanger sequencing was used for other members of the family and a control group of 500 healthy individuals. Bioinformatics analysis and three-dimensional structure predictions were used to predict the impact of amino acid changes on protein structure and function. Results. The candidate genes of cataract and iris coloboma were successfully screened out. A heterozygote mutation, CRYGD c.70C >A (p.P24T), was identified as cosegregating with congenital cataracts, while another heterozygous mutation, WFS1 c.1514G $>$ C (p.C505S), which had not been reported previously, cosegregated with congenital iris coloboma. Bioinformatic analyses and three-dimensional structure prediction proved that the three-dimensional structures of WFS1 p.C505S and CRYGD p.P24T changed markedly and may contribute significantly to iris coloboma and congenital cataract, respectively. Conclusions. We report a novel mutation, WFS1 p.C505S, and a known mutation, CRYGD p.P24T, that cosegregate with iris coloboma and congenital cataract, respectively, in a Chinese family. This is the first time the association of WFS1 p.C505S with iris coloboma has been demonstrated, although CRYGD p.P24T has been widely reported as being associated with congenital cataract, especially in the Eastern Asian population. These findings may have future therapeutic benefit for the diagnosis of iris coloboma and congenital cataract. The results may also be relevant in further studies aiming to investigate the molecular pathogenesis of iris coloboma and congenital cataract.

\section{Introduction}

Congenital cataract complicated by iris coloboma is not clinically common. Congenital cataract (OMIM 604307), which is an opacification of the ocular lens present at birth [1], is usually hereditary and can be transmitted as a dominant or a recessive trait. Congenital cataract is a major cause of visual impairment and blindness worldwide, with at least a third of which being familial, and accounts for $10 \%$ of all childhood blindness [2-4]. The primary consequences of lens cloudiness include glare, contrast loss, and decrease in vision that eventually lead to blindness. Various etiological 
factors have been identified, including infection, metabolic disorders, and genetic defects [5]. Iris coloboma is a special phenotype of congenital aniridia, defined as a rare bilateral preocular disorder characterized by the complete or partial absence of the iris [6]. Iris coloboma is most commonly inherited as an autosomal-dominant disorder [6]. It is an anatomical defect generated as a result of defective embryogenesis in ocular structures [7]. If the choroidal fissure is not completely closed at the iris, the defect will exist under the iris, and the round pupil will appear as a key hole shape known as iris coloboma. The prevalence range is approximately $1: 64,000$ to $1: 96,000$ worldwide, and approximately $1: 100,000$ in China [8].

Congenital cataracts are highly heterogeneous clinically and show interfamilial and intrafamilial variability [9]. At present, at least 45 genes of nonsyndromic congenital cataract with Mendelian inheritance have been reported [10-12]. (Table S1). The inheritance modes of these genes include autosomal dominant (AD), autosomal recessive (AR), and X-linked recessive (XR), but autosomal dominant is the main inheritance mode [13]. These pathogenic genes can be divided into six categories: lens protein gene, membrane protein gene, cytoskeleton protein gene, developmental regulatory protein gene, tyrosine kinase receptor gene, and chromatin-modified protein gene, in addition to some syndrome-related genes related to cataract [14]. Among them, lens protein genes, such as CRYAA, CRYAB, and $C R Y G D$, encode nearly $50 \%$ of the protein in the lens [14]. About two-thirds of the cases of congenital iris coloboma are inherited as an autosomal-dominant trait with genetic heterogeneity [15-17]. These genes have been mapped on chromosome $11 \mathrm{p} 13$ by linkage analysis and positional cloning. Approximately $80 \%$ of congenital iris coloboma cases are caused by the gene mutation of human paired box-6 (PAX6) [8].

This study aims to identify the pathogenic genes of cataract and iris coloboma in a family by whole exon sequencing combined with cosegregation analysis, identifying the mutation sites and performing informatic analysis to preliminarily detect the possible genetic factors of the disease, and to find strategies and methods for the treatment of cataract and iris coloboma.

\section{Materials and Methods}

2.1. Subject Recruitment and Clinical Examination. A family with congenital cataracts from a Han ethnicity was recruited for the present study. In total, 14 subjects were enrolled and underwent detailed clinical examinations. All members of the family were diagnosed by the Aier Eye Hospital in Hunan province. Four individuals were affected by cataracts, and three were diagnosed with iris coloboma with or without cataract. Peripheral blood samples were collected from all the survivors of the family after informed consent was obtained from all members. This study was approved by the Institutional Review Board of the Xiangya Hospital of Central South University (Changsha, China) and performed in accordance with the Declaration of Helsinki.
2.2. Genomic DNA Preparation and High Throughput Sequencing. The genomic DNA of peripheral blood lymphocytes of the probands was extracted by the phenol-chloroform method. DNA concentration and purity were detected by NanoDrop 1000 ultramicro spectrophotometer. Ultrasound degradation was used to break $5 \mu \mathrm{g}$ genomic DNA into 150-200 bp fragments, and junctions were added at both ends of the fragments to construct the library. The constructed library was amplified via polymerase chain reaction (PCR). The reaction system of library amplification was prepared on ice, and the reaction program was set up to carry out PCR on the PE-2720 PCR instrument. The thermal cycle conditions for PCR are as follows: initial denaturation at $95^{\circ} \mathrm{C}$ for $2 \mathrm{~min}$, followed by 30 cycles of denaturation at $95^{\circ} \mathrm{C}$ for $30 \mathrm{sec}$, annealing at $65^{\circ} \mathrm{C}$ for $30 \mathrm{sec}$ and elongation at $72^{\circ} \mathrm{C}$ for $1 \mathrm{~min}$, cycling 4-6 times, and a final extension at $75^{\circ} \mathrm{C}$ for $10 \mathrm{~min}$. The library capture mixture was prepared, the hybrids were captured, and the obtained hybrid library was further amplified by PCR. Finally, the hybrid library was sequenced by Illumina HiSeq 2000 system.

2.3. Bioinformatics Analysis. We evaluated the sequence quality and aligned the reads to the human reference genome (GRCh37/hg19) using a Burrows-Wheeler Aligner. Single nucleotide variations (SNVs) and insertions and deletions (Indels) were called by the genome analysis toolkit. The candidate variants were filtered according to the following rules: (1) eliminate variation in noncoding regions and synonymous variation in coding regions; (2) exclude nonrare (MAF $\geq 0.01$ ) variants in the 1000 Genomes Project and ESP Exon Project; (3) exclude the mutation in the sequencing data of the normal control exomes already in the laboratory.

2.4. Sanger Sequencing Analysis. In order to confirm whether the disease phenotype is cosegregated with candidate genes, Sanger sequencing was used to confirm that the two mutations were not detected in the unaffected family members and in another 500 unrelated controls. Peripheral blood was collected, and genomic DNA was extracted from blood lymphocytes and leukocytes by the standard phenol-chloroform method. Sanger sequencing of all the individuals was performed following the standard procedures.

2.5. Functional Prediction of Proteins. Twenty-four software programs and arithmetics, including SIFT, LRT, MutationTaster, FATHMM, PROVEAN, VEST3, MetaSVM, MetaLR, M-CAP, FATHMM-MKL, Eigen, GenoCanyon, fitCons, REVEL, ReVe, GERP++, phyloP, phastCons, SiPhy, CADD, DANN, MutationAssessor, PolyPhen-2 HVAR, and PolyPhen-2 HDIV in VarCards (http://159.226.67.237/sun/ varcards/welcome/index) [18], were employed to predict the harmfulness of mutated protein. I-TASSER (http://zhanglab. ccmb.med.umich.edu/I-TASSER/) and PyMol-1.5.x software were used to predict and visualize the three-dimensional (3D) model of the proteins. Additionally, the 3D 
model of reported mutants, which were in the same domain or in the same functional structures in the gene, was also predicted for contrastive analysis.

\section{Results}

3.1. Clinical Evaluation. A three-generation family with diagnosed cataract with iris coloboma was recruited for this study. The family consists of 14 individuals, including 6 males and 8 females (Figure 1). The slit lamp clinical examination of anterior segment revealed the proband (II:1) with congenital cataract accompanying iris coloboma (Figure 2). Patients I:2, II:1, II:5, and III:1 were diagnosed as having congenital cataracts, and patients I:2, II:1, and II:5 had accompanying iris coloboma simultaneously.

3.2. Mutation and Cosegregation Analyses. Sequence assembly with BWA and SAMtools was performed to obtain the genotype of each gene locus, and then detection and annotation classification of SNP and indels were performed by SAMtools and a genome analysis toolkit (GATK). Samples from the family were found to have 21,595 mutation sites containing 11,299 nonsynonymous mutations, splice junction mutations, and insertional deletion mutations. A total of 216 mutations with a frequency of less than 0.01 in the population were screened out from the 1000 Genomes database. Six mutation sites of five related genes (BCOR, FYCO1, SIX6, WFS1, and CRYGD) were screened by comparison with data provided by ClinVar.

A heterozygous mutation c.70C $>\mathrm{A}$ in the $C R Y G D$ gene and a heterozygous mutation c.1514G $>\mathrm{C}$ in the WFS1 gene were detected in the proband II:1 (Figure 3). In addition, the CRYGD c.70C>A mutation was screened out in another three cataract patients (I:2, II:5 and III:1), and WFS1 c. $1514 \mathrm{G}>\mathrm{C}$ was screened out in another two iris coloboma patients (I:2, and II:5) (Figure 1). Neither CRYGD c.70C>A nor WFS1 c.1514G>C was detected in the healthy individuals in this lineage or in the other 500 healthy patients. It was thus confirmed that CRYGD c.70C $>\mathrm{A}$ and WFS1 c.1514G $>\mathrm{C}$ cosegregate with congenital cataract and iris coloboma, respectively.

3.3. Bioinformatics Analysis. Because the heterozygous c.70C > T (p.P24T) in CRYGD has been recognized as the cataract-causing gene mutation, $[2,19-24]$ we performed functional prediction only for WFS1 c.1514G>C. In our results, 19 of the 24 functional prediction programs in VarCards showed that c.1514G $>$ C in WFS1 was possibly harmful (Table 1).

3D structure prediction software was used to reveal the difference between the wild type and the mutant type in CRYGD and WFS1. The wild type of CRYGD p.P24 exhibited a pyrrole ring structure, while the p.P24T mutant changed into a hydrophilic structure (Figure 4). We studied another six reported mutation sites located in the first "Greek Key" module domain of CRYGD protein as having the p.P24T mutant, such as p.R15C, p.R15S, p.P24S, p.A36P, p.R37P, and p.R37S, which have been reported to be associated with cataracts. According to the results of 3D structure prediction, compared with the wild type CRYGD, all the three mutants, p.R15C, p.R15S, and p.R37S, changed structure noticeably from the long side to the short side chains, while p.A36P and p.R37P exhibited proline with pyrrole rings in comparison to the wild type. However, in mutants p.P24S and p.P24T the original pyrrole rings did not appear in the wild type protein (Figure 4).

Moreover, three reported disease-causing mutations in WFS1, including p.G437R, p.S443I, and p.P504L, located in the transmembrane domain as with p.C505S, were 3D structures predicted as requiring further analysis. In that analysis, p.P504L did not exhibit original pyrrole rings compared with p.P504, while p.G437R, p.S443I, and p.P504L underwent much greater changes than the wild type (Figure 5).

\section{Discussion}

With the development of exon sequencing technology and its decreasing cost, it has been widely used in screening for disease-causing genes. In this study, the candidate genes for cataract and iris coloboma were successfully screened by exon sequencing in the probands with cataract and iris coloboma. By Sanger sequencing, a heterozygote mutation, c.70C > A (p.P24T) in CRYGD, was screened out in all cataract patients and was not detected in noncataract patients. A heterozygous mutation, c.1514G $>$ C (p.C505S) in WFS1, was screened in all iris coloboma patients and was absent in subjects without iris coloboma. In addition, these mutations were absent in the other 500 random healthy individuals. It was thus confirmed that CRYGD c.70C>A and WFS1 c.1514G $>C$ cosegregate with the disease phenotypes examined. It is also preliminarily confirmed that these two gene mutations are responsible for congenital cataract and iris coloboma, respectively.

Literature review shows that cataracts caused by the mutation in CRYGD had been reported in many published articles since the 1990s and that the biological function of the mutant protein was deeply and meticulously researched in many papers $[2,21,22,25-27]$. The p.P24T mutation in $C R Y G D$ has been widely reported as the cause of congenital cataract, especially in the East Asian population [2, 19-24]. The CRYGD gene encodes $\gamma \mathrm{D}$-crystallin, the lens protein, with 174 amino acids $[28,29] . \gamma \mathrm{D}$-crystallins are monomer proteins whose polypeptide chains fold into four similar "Greek key" motifs. The last two of the "Greek key" modules form a domain joined by connecting fragments [30,31]. At least eight mutations related to congenital cataract have been reported in the lens protein gene CRYGD $[2,21,22,26,27,32]$. These mutations can change the structure or three-dimensional conformation of lens protein, affecting its solubility, stability, or orderliness, leading to cataracts $[2,21,22,26,27]$. In this study, we analyzed the structure of p.P24T and another six mutation sites in the first "Greek Key" module domain of the CRYGD protein, that is, p.R15C, p.R15S, p.P24S, p.A36P, p.R37S, and p.R37P, which have been reported as being associated with cataract.

I-TASSER and PyMol-1.5.x software were used to investigate the $3 \mathrm{D}$ structure of the protein and to compare wild 


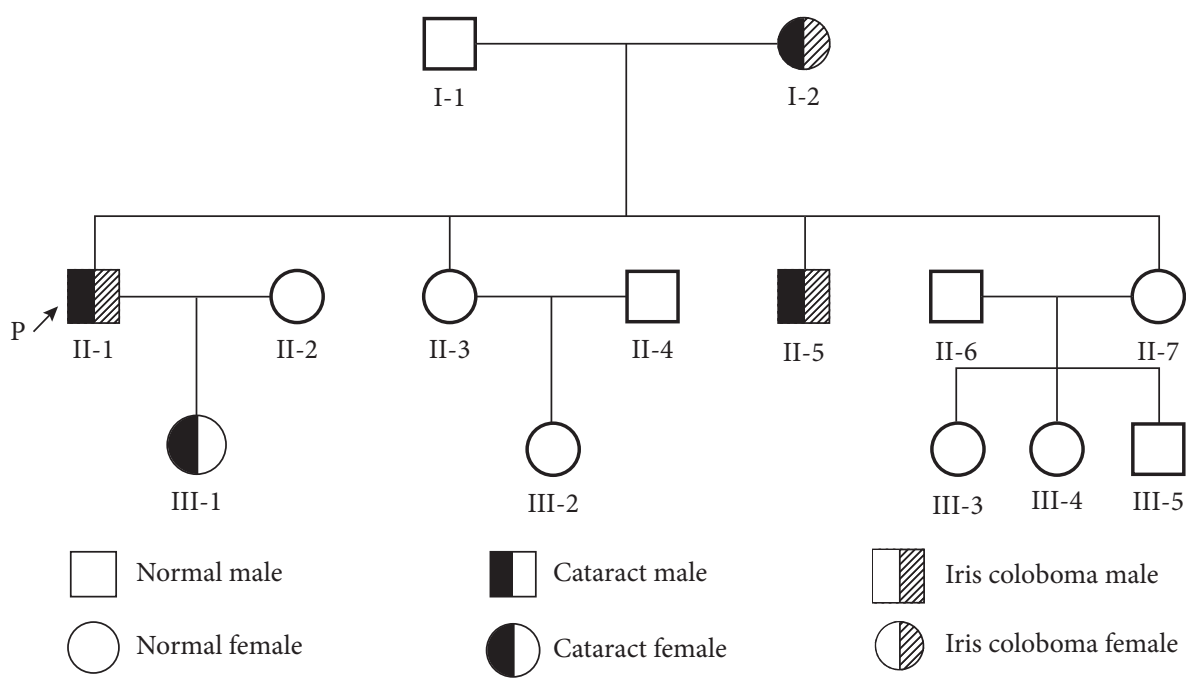

Figure 1: Family diagram. The pedigree of the congenital cataract with iris coloboma: circles represent women; squares represent men. Black indicates cataract patients, gray shadows indicate iris coloboma patients, and the arrow indicates the proband (II:1).

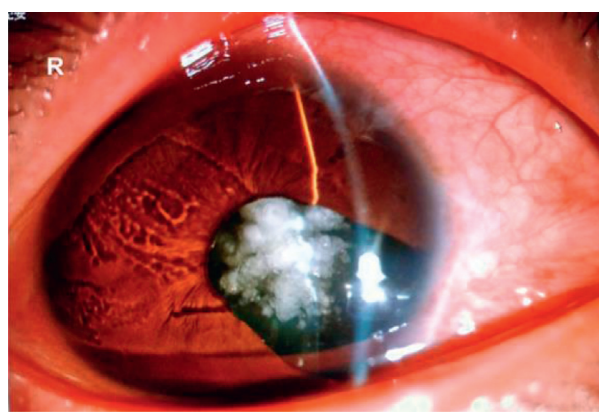

(a)

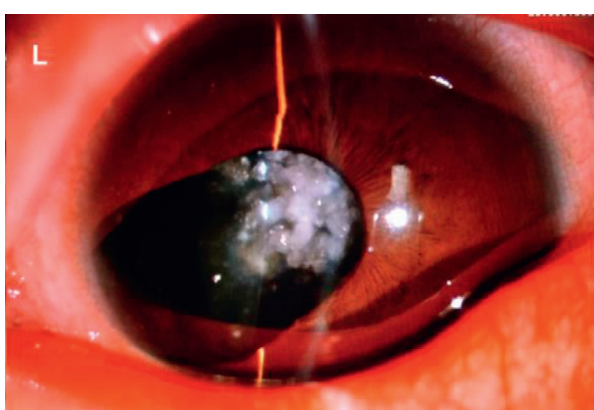

(b)

FIGURE 2: Proband (II:1). Photograph of the anterior segment of the slit lamp. It can be seen that the bilateral cornea of the patient is clear, the pupil is not round, the iris is missing, and the central cauliflower pattern is cloudy.

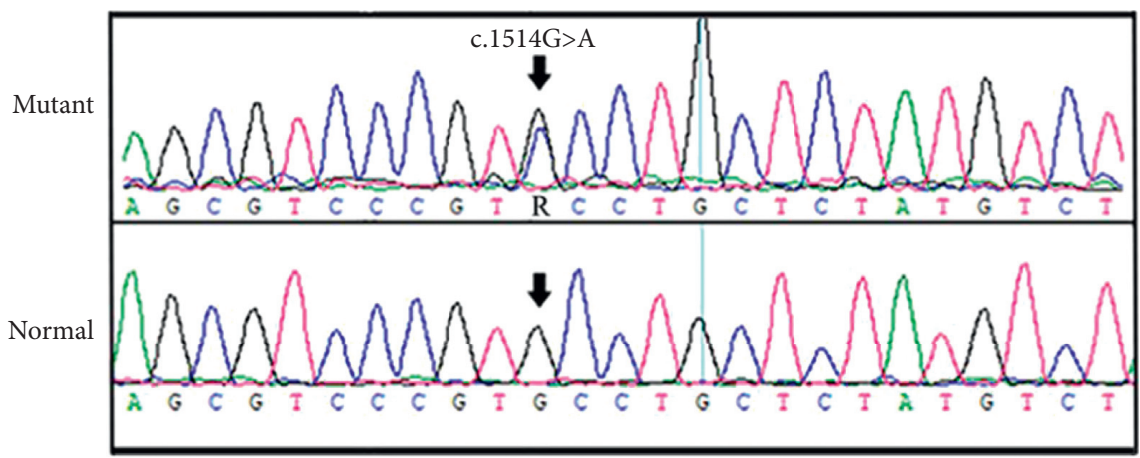

(a)

Figure 3: Continued. 


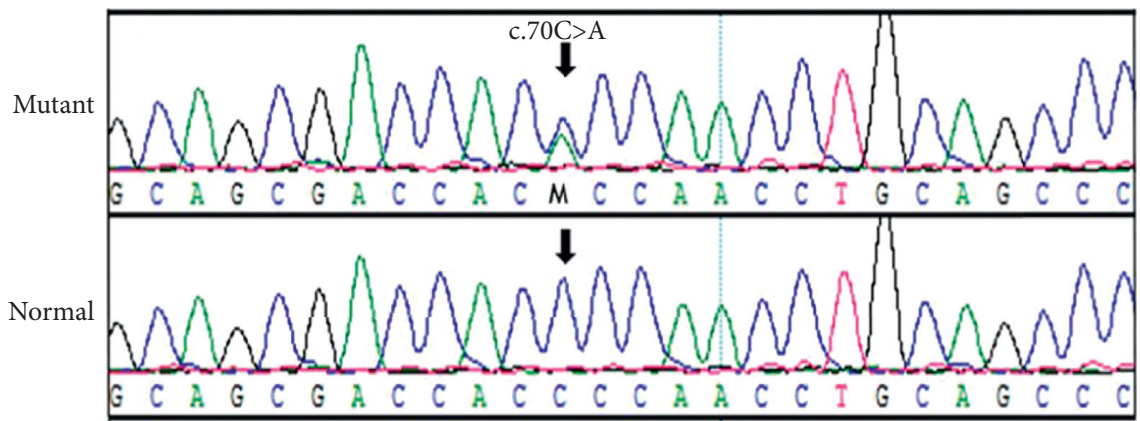

(b)

FiguRe 3: Mutant screening sequencing alignment diagram for family. (a) The sequence chromatogram (forward strand) shows a heterozygous c.1514G $>$ A transition that causes a substitution of serine (S) for cysteine (C) at codon 505 . The arrow points to the position of the mutant nucleotide. (b) The sequence chromatogram (forward strand) shows a heterozygous c.70C $>$ T transition that causes a substitution of threonine $(\mathrm{T})$ for proline $(\mathrm{P})$ at codon 24 . The arrow points to the position of the mutant nucleotide.

TABLE 1: Functional prediction results by twenty-four software programs and arithmetics.

\begin{tabular}{lc}
\hline Gene symbol & WFS1 \\
\hline Nucleotide change & c. 1514G>C \\
Exon & 8 \\
Amino acid change & p.C505S \\
Domain & Fifth transmembrane domain \\
SIFT & Damaging \\
LRT & Deleterious \\
Mutation taster & Disease-causing \\
FATHMM & Damaging \\
PROVEAN & Damaging \\
VEST3 & Damaging \\
MetaSVM & Damaging \\
MetaLR & Damaging \\
M-CAP & Damaging \\
FATHMM-MKL & Damaging \\
Eigen & Damaging \\
GenoCanyon & Damaging \\
fitCons & Damaging \\
REVEL & Damaging \\
ReVe & Damaging \\
GERP++ & Conserved \\
phyloP & Conserved \\
phastCons & Conserved \\
SiPhy & Conserved \\
CADD & Tolerable \\
DANN & Tolerable \\
MutationAssessor & Medium \\
PolyPhen-2 HDIV & Benign \\
PolyPhen-2 HVAR & Benign \\
\hline
\end{tabular}

type CRYGD with the p.P24T mutant. It was revealed that the substitution of proline with threonine changes the structure of the amino acid at position 24 from a pyrrole ring to a nonpyrrole ring (Figure 4). After proline was changed to threonine, there was a branched beta carbon atom in the side chain of the threonine, making the beta-chain tend to extend rather than fold back, which may affect the correct folding of the protein material $[2,33]$. Previous studies have shown that the solubility of the p.P24T mutant protein is significantly lower than that of the wild type CRYGD protein, which may lead to decreased solubility and the aggregation of polymer material $[2,33]$.

The p.R15C mutation is located in exon 2 of the CRYGD gene and changes a hydrophilic charged amino acid (arginine) to a neutral one (cysteine) (Figure 4). Previous studies showed that the presence of newly exposed cysteine residues in p.R15C mutants would allow the formation of intermolecular disulfide bonds under oxidative conditions, leading to protein aggregation, which is the main feature of many cataracts [34]. In addition, increased hydrophobicity at the p.R15S mutation site may affect protein-protein interactions, since p.R15 is located on a solvent-accessible protein surface [35]. Moreover, the change of p.P24S from hydrophobic proline with pyrrole ring to serine with hydrophilic structure may affect the solubility of $\gamma \mathrm{D}$-crystallin $[19,20,36]$. This effect may be caused by a change in the hydrogen binding characteristics of the protein-water interface $[19,20,36]$. Regarding p.A36P, it was reported that the mutant structure may affect the normal folding in the tertiary structure of amino acids, leading to decreased solubility, and the accumulation of macromolecule substances may lead to decreased lens transparency, eventually leading to the occurrence of cataract [37]. Moreover, previous studies showed that p.P37S may lead to decreased solubility, and serine residues will destroy the normal beta lamellar structure of proteins, allowing the formation of "beta-bulge" [38], while p.R37P reduced protein solubility by increasing the local hydrophobicity in affected individuals [39].

Congenital iris coloboma is caused by incomplete closure of the fetal cleft at organogenesis [7]. Colobomas of the iris are closure defects of embryonic fissures that can occur in the fifth week of gestation. They often appear in the lower nasal quadrant and can be either isolated or associated with colobomas of the lens, choroid, and optic nerve [40, 41]. Prior to this study, WFS1 p.C505S had not been reported in association with the iris coloboma phenotype. The WFS1 gene encodes the transmembrane protein (wolframin) with 890 amino acids containing nine transmembrane domains [42]. WFS1 was known as the main pathogenic gene of Wolfram syndrome [43], which is an autosomal recessive 


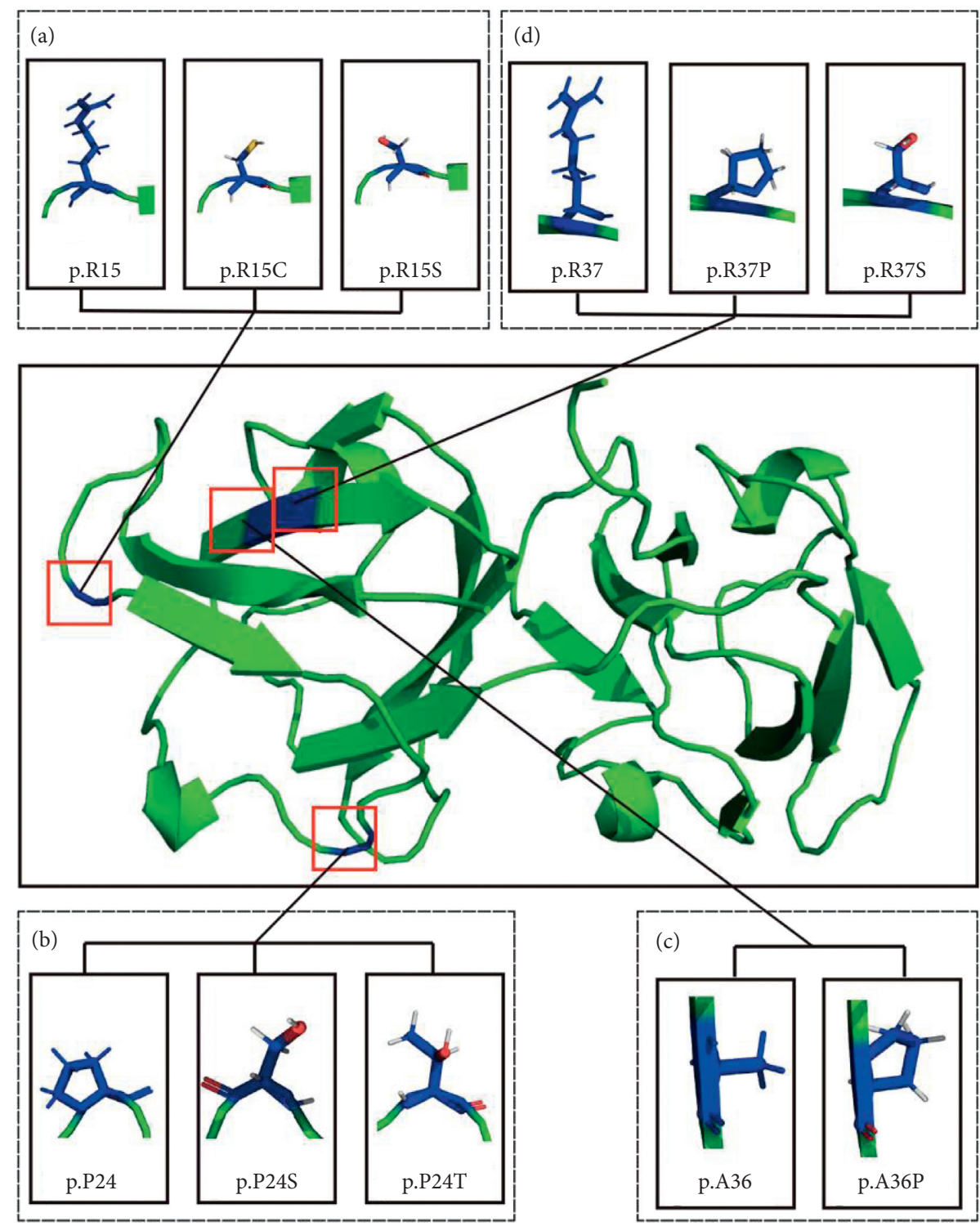

Figure 4: The 3D conformation of seven reported pathogenic mutation sites located in the first "Greek Key" module domain of CRYGD, as predicted by modeling software. (a) The 3D conformation of the wild type CRYGD 15th amino acid arginine and mutant cysteine and serine. (b) The 3D conformation of the wild type CRYGD 24th amino acid proline and the mutant threonine and serine. (c) The 3D conformation of the wild type CRYGD 36th amino acid alanine and the mutant Proline. (d) The 3D conformation of the wild type CRYGD 37th amino acid arginine and mutant serine and proline. 3D, three-dimensional.

neurodegenerative disorder characterized by visual symptoms, diabetes mellitus, central diabetes insipidus, and deafness [44]. WFS1 is also a key element in the endoplasmic reticulum stress activation signaling cascade known as unfolded protein response [45]. Therefore, the mutation of WFS1 may affect wolframin in regulating the homeostasis of endoplasmic reticulum, thereby causing incomplete closure of the fetal fissure during organogenesis, resulting in iris coloboma. Studies show that changes in the transmembrane domain have a serious impact on the function of wolframin proteins [46]. However, the mutation p.C505S was located in the fifth domain. For further comparison and analysis, p.C505S and three other missense mutations reported to cause disease were predicted by $3 \mathrm{D}$ structures in I-TASSER.
The predicted results from 15 functional prediction software programs showed c.1514G $>\mathrm{C}$ in WFS1 was possibly harmful. The novel p.C505S mutation is likely pathogenic for the following reasons: (1) it has never been reported, and it achieved cosegregation with disease phenotypes; (2) it occurs within the highly preserved fifth transmembrane segment, and it is close to the p.P504L mutation, which was previously reported as a diseasecausing gene [42]. 3D structure prediction exhibited that p.G437R, p.S443I, and p.P504L underwent greater change than the wild type. The p.G437R mutation, which changed from a neutral hydrophobic glycine to an alkaline hydrophilic arginine, was reported to destroy the normal function of wolframin and lead to Wolfram syndrome in 


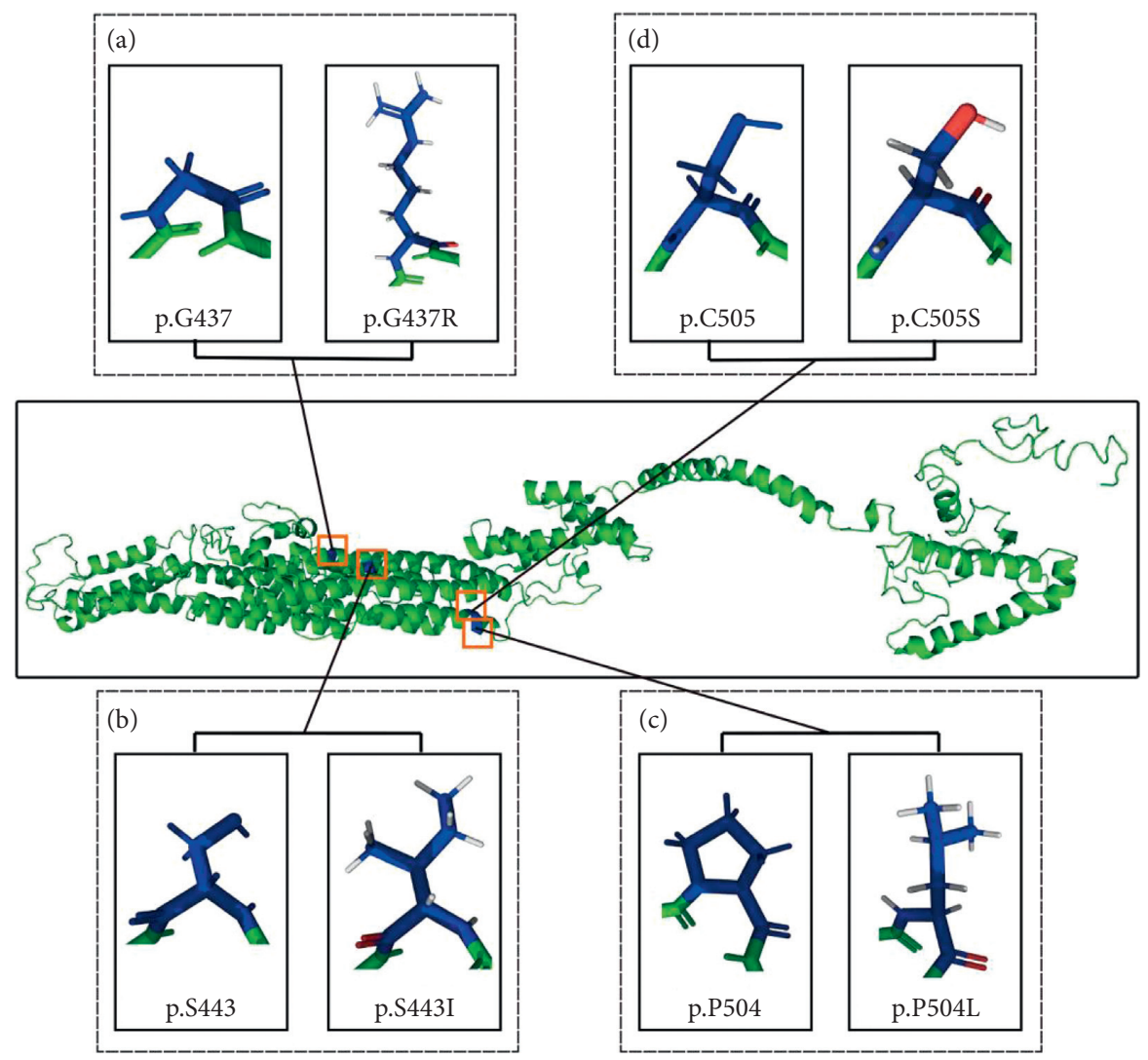

FIGURE 5: The 3D conformation of four mutation sites located in the transmembrane domain of WFS1, as predicted by modeling software. (a) The 3D conformation of the wild type WFS1 437th amino acid glycine and mutant arginine. (b) The 3D conformation of the wild type WFS1 443rd amino acid serine and the mutant isoleucine. (c) The 3D conformation of the wild type WFS1 504th amino acid proline and the mutant leucine. (d) The 3D conformation of the wild type WFS1 505th amino acid cysteine and mutant serine. 3D, three-dimensional.

Great Britain [46]. By contrast, p.S443I changed a hydrophilic amino acid into a hydrophobic one. Studies showed that the polarity change caused by p.S443I mutation is likely to interrupt the transmembrane domains of wolframin [47]. Compared to p.P504, the 3D structure of p.P504L exhibited proline with a pyrrole ring to a leucine with nonpyrrole ring (Figure 5). Studies showed that the p.P504L mutation is associated with the onset of Wolfram syndrome [48]. In addition, the $648^{\text {th }}$ amino acid, a reported disease-causing gene located on the transmembrane, was deemed to be an easily truncated variant $[46,49]$.

Above all, our research provided a reference for the diagnosis of congenital iris coloboma. Meanwhile, we firstly described WFS1 gene mutation associated with congenital iris coloboma and enriched its gene mutation spectrum, which may also provide a new sight for gene therapy for this disease.

However, our study does have several limitations. First, the sample size was relatively small, only the Chinese population was screened, and, compared with other small size group research, our description of family history and clinical symptoms is not comprehensive enough [50-52]. In addition, some research indicated that the mutation of WFS1 may also cause cataract, so we cannot exclude the possibility that the joint action of the CRYGD and WFS1 cause cataract and iris coloboma [37, 53, 54]. Second, although some software has been used to predict mutations, it has not been validated by molecular, cellular, and animal experiments. Finally, although we have done a lot of research on the WFS1 protein, the exact molecular function of the WFS1 protein in the development of the iris remains unknown.

\section{Conclusion}

In conclusion, a novel WFS1 p.C505S mutation and a CRYGD p.P24T mutation were found in a family with cataract and iris coloboma, and they were cosegregated from iris coloboma and cataract, respectively. Structural analysis in this study also highlighted the unique role of a single amino acid in the change of protein properties. It was proved that the C505S mutation of WFS1 contributes greatly to the occurrence of iris coloboma and corroborated that the CRYGD p.P24T possibly leads to cataract, although CRYGD p.P24T has been detected in East Asia widely and identified as having a biological function in cataracts. Further exploration of the pathogenesis of the disease is needed, as it also plays a major role in prevention and treatment of the disease. We expect that more researchers will take up exon sequencing combined with bioinformatics analysis as a major scientific method and diagnostic tool. 


\section{Data Availability}

The data used to support the findings of this study are included within the article.

\section{Conflicts of Interest}

The authors declare that they have no conflicts of interest.

\section{Authors' Contributions}

Bin Li and Bin Lu contributed equally to this work.

\section{Acknowledgments}

The authors are grateful to the colleagues for their participation in these studies. The present study was supported by the Natural Science Foundation of Hunan Province, China (No. 2017JJ3507), and by the Postdoctoral Science Foundation of Xiangya Hospital, Central South University, China (No. 175817).

\section{Supplementary Materials}

Table S1 reports 45 genes of nonsyndromic congenital cataract with Mendelian inheritance. Genes with widely reported mutations that are associated congenital cataract were listed with gene symbol, nucleotide change, amino acid change, clinical phenotype, and reference. (Supplementary Materials)

\section{References}

[1] E. Liu and K. Kopani, "Rapidly progressive cataract formation associated with non-small-cell lung cancer therapy," Journal of Cataract and Refractive Surgery, vol. 42, no. 12, pp. 18381840, 2016.

[2] J. C. Boatz, M. J. Whitley, M. Li et al., "Cataract-associated $\mathrm{P} 23 \mathrm{~T}$ gammaD-crystallin retains a native-like fold in amorphous-looking aggregates formed at physiological $\mathrm{pH}$," $\mathrm{Na}$ ture Communications, vol. 8, p. 15137, 2017.

[3] A. L. Solebo, C. J. Hammond, and J. S. Rahi, "Improving outcomes in congenital cataract," Nature, vol. 556, no. 7699, pp. E1-E2, 2018.

[4] C. L. Chen, G. A. Lin, N. S. Bardach et al., "Preoperative medical testing in Medicare patients undergoing cataract surgery," New England Journal of Medicine, vol. 372, no. 16, pp. 1530-1538, 2015.

[5] S. W. Nam, D. H. Lim, K. Y. Cho et al., "Risk factors of presenile nuclear cataract in health screening study," $B M C$ Ophthalmology, vol. 18, no. 1, p. 263, 2018.

[6] H. Lee, R. Khan, and M. O’Keefe, "Aniridia: current pathology and management," Acta Ophthalmologica, vol. 86, no. 7, pp. 708-715, 2008.

[7] N. Zerkaoui, "Bilateral iris coloboma," Pan African Medical Journal, vol. 30, p. 1, 2018.

[8] J. J. Qiu, Q. Zhang, Z. X. Geng et al., "Identification of a novel PAX6 mutation in a Chinese family with aniridia," $B M C$ Ophthalmology, vol. 19, no. 1, p. 10, 2019.

[9] E. Yonova-Doing, Z. A. Forkin, P. G. Hysi et al., "Genetic and dietary factors influencing the progression of nuclear cataract," Ophthalmology, vol. 123, no. 6, pp. 1237-1244, 2016.
[10] L. Yuan, Y. Guo, J. Yi et al., "Identification of a novel GJA3 mutation in congenital nuclear cataract," Optometry and Vision Science, vol. 92, no. 3, pp. 337-342, 2015.

[11] H. Wang, T. Zhang, D. Wu et al., "A novel beaded filament structural protein 1 (BFSP1) gene mutation associated with autosomal dominant congenital cataract in a Chinese family," Molecular Vision, vol. 19, pp. 2590-2595, 2013.

[12] X. Y. Xia, N. Li, X. Cao et al., "A novel COL4A1 gene mutation results in autosomal dominant non-syndromic congenital cataract in a Chinese family," BMC Medical Genetics, vol. 15, no. 1, p. 97, 2014.

[13] H. Deng and L. Yuan, "Molecular genetics of congenital nuclear cataract," European Journal of Medical Genetics, vol. 57, no. 2-3, pp. 113-122, 2014.

[14] A. S. Ma, J. R. Grigg, G. Ho et al., "Sporadic and familial congenital cataracts: mutational spectrum and new diagnoses using next-generation sequencing," Human Mutation, vol. 37, no. 4, pp. 371-384, 2016.

[15] J. Prosser and V. van Heyningen, "PAX6 mutations reviewed," Human Mutation, vol. 11, no. 2, pp. 93-108, 1998.

[16] A. Kondo-Saitoh, N. Matsumoto, T. Sasaki et al., “Two nonsense mutations of PAX6 in two Japanese aniridia families: case report and review of the literature," European Journal of Ophthalmology, vol. 10, no. 2, pp. 167-172, 2000.

[17] C. Jin, Q. Wang, J. Li et al., "A recurrent PAX6 mutation is associated with aniridia and congenital progressive cataract in a Chinese family," Molecular Vision, vol. 18, pp. 465-470, 2012.

[18] J. Li, L. Shi, K. Zhang et al., "VarCards: an integrated genetic and clinical database for coding variants in the human genome," Nucleic Acids Research, vol. 46, no. D1, pp. D1039D1048, 2018.

[19] P. Evans, K. Wyatt, G. J. Wistow, O. A. Bateman, B. A. Wallace, and C. Slingsby, "The P23T cataract mutation causes loss of solubility of folded $\gamma$ d-crystallin," Journal of Molecular Biology, vol. 343, no. 2, pp. 435-444, 2004.

[20] A. Pande, O. Annunziata, N. Asherie, O. Ogun, G. B. Benedek, and J. Pande, "Decrease in protein solubility and cataract formation caused by the Pro23 to thr mutation in human $\gamma \mathrm{d}$ crystallin," Biochemistry, vol. 44, no. 7, pp. 2491-2500, 2005.

[21] G. Yang, Z. Chen, W. Zhang et al., "Novel mutations in CRYGD are associated with congenital cataracts in Chinese families," Scientific Reports, vol. 6, p. 18912, 2016.

[22] J. Li, Y. Leng, S. Han et al., "Clinical and genetic characteristics of Chinese patients with familial or sporadic pediatric cataract," Orphanet Journal of Rare Diseases, vol. 13, no. 1, p. 94, 2018.

[23] A. Soares, A. Mota, S. Fonseca et al., "Ophthalmologic manifestations of Wolfram syndrome: report of 14 cases," Ophthalmologica, pp. 1-4, 2018.

[24] F. Ji, L. M. I. Koharudin, J. Jung, and A. M. Gronenborn, "Crystal structure of the cataract-causing P23T $\gamma$ D-crystallin mutant," Proteins: Structure, Function, and Bioinformatics, vol. 81, no. 9, pp. 1493-1498, 2013.

[25] X. Zhuang, L. Wang, Z. Song et al., "A novel insertion variant of CRYGD is associated with congenital nuclear cataract in a Chinese family," PLoS One, vol. 10, no. 7, Article ID e0131471, 2015.

[26] M. Gao, S. Huang, J. Li et al., "[A novel pathogenic mutation of CRYGD gene in a congenital cataract family]," Zhonghua Yi Xue Yi Chuan Xue Za Zhi, vol. 33, no. 4, pp. 515-518, 2016.

[27] L. Dudakova, V. Stranecky, O. Ulmanova et al., "Segregation of a novel p.(Ser270Tyr) MAF mutation and p.(Tyr56*) CRYGD variant in a family with dominantly inherited 
congenital cataracts," Molecular Biology Reports, vol. 44, no. 6, pp. 435-440, 2017.

[28] A. Pande, J. Pande, N. Asherie et al., "Molecular basis of a progressive juvenile-onset hereditary cataract," Proceedings of the National Academy of Sciences, vol. 97, no. 5, pp. 19931998, 2000.

[29] S. Garcia-Manyes, D. Giganti, C. L. Badilla et al., "Singlemolecule force spectroscopy predicts a misfolded, domainswapped conformation in human $\gamma$ d-crystallin protein," Journal of Biological Chemistry, vol. 291, no. 8, pp. 4226-4235, 2016.

[30] E. Serebryany, T. Takata, E. Erickson, N. Schafheimer, Y. Wang, and J. A. King, "Aggregation of $\operatorname{Trp}>$ Glu point mutants of human gamma-D crystallin provides a model for hereditary or UV-induced cataract," Protein Science, vol. 25, no. 6, pp. 1115-1128, 2016.

[31] E. Serebryany, J. C. Woodard, B. V. Adkar, M. Shabab, J. A. King, and E. I. Shakhnovich, "An internal disulfide locks a misfolded aggregation-prone intermediate in cataractlinked mutants of human $\gamma \mathrm{D}$-crystallin," Journal of Biological Chemistry, vol. 291, no. 36, pp. 19172-19183, 2016.

[32] C. Ma, G. Zheng, and L. Hao, "Analysis of disease-causing gene mutation in three Chinese families with congenital inherited cataract," Zhonghua Yi Xue Yi Chuan Xue Za Zhi, vol. 35, no. 2, pp. 165-168, 2018.

[33] D. S. Mackay, T. M. Bennett, S. M. Culican et al., "Exome sequencing identifies novel and recurrent mutations in GJA8 and CRYGD associated with inherited cataract," Human Genomics, vol. 8, p. 19, 2014.

[34] D. A. Stephan, E. Gillanders, D. Vanderveen et al., "Progressive juvenile-onset punctate cataracts caused by mutation of the D-crystallin gene," Proceedings of the National Academy of Sciences, vol. 96, no. 3, pp. 1008-1012, 1999.

[35] L. Y. Zhang, B. Gong, J. P. Tong et al., "A novel gammaDcrystallin mutation causes mild changes in protein properties but leads to congenital coralliform cataract," Molecular Vision, vol. 15, pp. 1521-1529, 2009.

[36] O. V. Plotnikova, F. A. Kondrashov, P. K. Vlasov, A. P. Grigorenko, E. K. Ginter, and E. I. Rogaev, "Conversion and compensatory evolution of the $\gamma$-crystallin genes and identification of a cataractogenic mutation that reverses the sequence of the human CRYGD gene to an ancestral state," The American Journal of Human Genetics, vol. 81, no. 1, pp. 32-43, 2007.

[37] W. Sun, X. Xiao, S. Li et al., "Mutation analysis of 12 genes in Chinese families with congenital cataracts," Molecular Vision, vol. 17, pp. 2197-2206, 2011.

[38] A. Pande, J. Pande, N. Asherie et al., "Crystal cataracts: human genetic cataract caused by protein crystallization," Proceedings of the National Academy of Sciences, vol. 98, no. 11, pp. 6116-6120, 2001.

[39] L. Wang, X. Chen, Y. Lu et al., "A novel mutation in gammaDcrystallin associated with autosomal dominant congenital cataract in a Chinese family," Molecular Vision, vol. 17, pp. 804-809, 2011

[40] R. Ozelce, V. Gurlu, H. Guclu et al., "Coexistence of optic pit and coloboma of iris, lens, and choroid: a case report," Arquivos Brasileiros de Oftalmologia, vol. 79, no. 5, pp. 328-329, 2016.

[41] A. S. Karatepe Hashas, H. B. Arifoglu, Y. Yuce et al., "Evaluations of corneas in eyes with isolated Iris coloboma," Current Eye Research, vol. 42, no. 1, pp. 41-46, 2017.

[42] V. Bansal, B. O. Boehm, and A. Darvasi, "Identification of a missense variant in the WFS1 gene that causes a mild form of
Wolfram syndrome and is associated with risk for type 2 diabetes in Ashkenazi Jewish individuals," Diabetologia, vol. 61, no. 10, pp. 2180-2188, 2018.

[43] M. L. de Heredia, R. Clèries, and V. Nunes, "Genotypic classification of patients with Wolfram syndrome: insights into the natural history of the disease and correlation with phenotype," Genetics in Medicine, vol. 15, no. 7, pp. 497-506, 2013.

[44] L. Rigoli, P. Bramanti, C. Di Bella, and F. De Luca, "Genetic and clinical aspects of Wolfram syndrome 1, a severe neurodegenerative disease," Pediatric Research, vol. 83, no. 5, pp. 921-929, 2018.

[45] S. Ghirardello, E. Dusi, B. Castiglione et al., "Congenital central diabetes insipidus and optic atrophy in a Wolfram newborn: is there a role for WFS1 gene in neurodevelopment?" Italian Journal of Pediatrics, vol. 40, p. 76, 2014.

[46] C. Hardy, F. Khanim, R. Torres et al., "Clinical and molecular genetic analysis of 19 Wolfram syndrome kindreds demonstrating a wide spectrum of mutations in WFS1," The American Journal of Human Genetics, vol. 65, no. 5, pp. 1279-1290, 1999.

[47] A. Tessa, I. Carbone, M. C. Matteoli et al., "Identification of novel WFS1 mutations in Italian children with Wolfram syndrome," Human Mutation, vol. 17, no. 4, pp. 348-349, 2001.

[48] F. Giuliano, S. Bannwarth, S. Monnot et al., "Wolfram syndrome in French population: characterization of novel mutations and polymorphisms in theWFS1 gene," Human Mutation, vol. 25, no. 1, pp. 99-100, 2005.

[49] H. Inoue, Y. Tanizawa, J. Wasson et al., "A gene encoding a transmembrane protein is mutated in patients with diabetes mellitus and optic atrophy (Wolfram syndrome)," Nature Genetics, vol. 20, no. 2, pp. 143-148, 1998.

[50] L. Duan, Q. Li, A. L. Tong et al., "Clinical characteristics of Wolfram syndrome in Chinese population and a novel frameshift mutation in WFS1," Frontiers in Endocrinology, vol. 9, p. 18, 2018.

[51] E. Ferro, A. P. Capra, G. Zirilli et al., "FTL c.-168G $>$ C mutation in hereditary hyperferritinemia cataract syndrome: a new Italian family," Pediatric and Developmental Pathology, vol. 21, no. 5, pp. 456-460, 2018.

[52] G. Çelmeli, D. Türkkahraman, Y. Çürek, J. Houghton, S. Akçurin, and İ. Bircan, "Clinical and molecular genetic analysis in three children with Wolfram syndrome: a novel WFS1 mutation (c.2534T > A)," Journal of Clinical Research in Pediatric Endocrinology, vol. 9, no. 1, pp. 80-84, 2017.

[53] V. Berry, C. Gregory-Evans, W. Emmett et al., "Wolfram gene (WFS1) mutation causes autosomal dominant congenital nuclear cataract in humans," European Journal of Human Genetics, vol. 21, no. 12, pp. 1356-1360, 2013.

[54] Z. Wang, C. Huang, Y. Sun et al., "Novel mutations associated with autosomal-dominant congenital cataract identified in Chinese families," Experimental and Therapeutic Medicine, vol. 18, no. 4, pp. 2701-2710, 2019. 